\title{
Association of Brain Lesions and Videofluoroscopic Dysphagia Scale Parameters on Patients With Acute Cerebral Infarctions
}

\author{
Sang Jun Mo, $\mathrm{MD}^{1}$, Ho Joong Jeong, $\mathrm{MD}, \mathrm{PhD}^{2}$, Yong Hyun Han, $\mathrm{MD}^{1}$, \\ Kihun Hwang, $\mathrm{MD}^{1}$, Jong Kyoung Choi, $\mathrm{MD}^{1}$ \\ ${ }^{1}$ Department of Rehabilitation Medicine, Dong-Eui Medical Center, Busan; \\ ${ }^{2}$ Department of Physical Medicine and Rehabilitation, Kosin University College of Medicine, Busan, Korea
}

Objective To investigate the characteristics and risk factors of dysphagia using the videofluoroscopic dysphagia scale (VDS) with a videofluoroscopic swallowing study (VFSS) in patients with acute cerebral infarctions.

Methods In this retrospective study, the baseline VFSS in 275 stroke patients was analyzed. We divided patients into 8 groups according to lesion areas commonly observed on brain magnetic resonance imaging. Dysphagia characteristics and severity were evaluated using the VDS. We also analyzed the relationship between clinical and functional parameters based on medical records and VDS scores.

Results In comparison studies of lesions associated with swallowing dysfunction, several groups with significant differences were identified. Apraxia was more closely associated with cortical middle cerebral artery territory lesions. Vallecular and pyriform sinus residue was more common with lesions in the medulla or pons. In addition, the results for the Korean version of the Modified Barthel Index (K-MBI), a functional assessment tool, corresponded to those in the quantitative evaluation of swallowing dysfunctions.

Conclusion A large cohort of patients with cerebral infarction was evaluated to determine the association between brain lesions and swallowing dysfunction. The results can be used to establish a specific treatment plan. In addition, the characteristic factors associated with swallowing dysfunctions were also confirmed.

Keywords Deglutition disorders, Deglutition, Fluoroscopy, Cerebral infarction

\section{INTRODUCTION}

Normal swallowing is a complex and sequential pro- cess that encompasses oral, pharyngeal, and esophageal phases. Defects in this process cause dysphagia. Dysphagia is particularly common when associated with brain

\footnotetext{
Received September 6, 2017; Accepted October 18, 2017

Corresponding author: Ho Joong Jeong

Department of Physical Medicine and Rehabilitation, Kosin University College of Medicine, 262 Gamcheon-ro, Seo-gu, Busan 49267, Korea. Tel: +8251-990-6156, Fax: +82-51-241-2019, E-mail: jhjpmr@naver.com

ORCID: Sang Jun Mo (http://orcid.org/0000-0003-1861-4636); Ho Joong Jeong (http://orcid.org/0000-0002-0607-2799); Yong Hyun Han (http://orcid. org/0000-0002-8960-186X); Kihun Hwang (http://orcid.org/0000-0002-8041-4363); Jong Kyoung Choi (http://orcid.org/0000-0002-9141-2013).

(c) This is an open-access article distributed under the terms of the Creative Commons Attribution Non-Commercial License (http://creativecommons.org/ licenses/by-nc/4.0) which permits unrestricted noncommercial use, distribution, and reproduction in any medium, provided the original work is properly cited. Copyright ( 2018 by Korean Academy of Rehabilitation Medicine
} 
lesions, and is found in approximately $50 \%$ of stroke patients [1]. If persistent, dysphagia causes nutritional deficiencies and dehydration. Retained food in the mouth, pharynx, and esophagus can lead to aspiration pneumonia and sepsis [1-3]. Therefore, when dysphagia occurs in stroke patients, oral and pharyngeal function must be evaluated as soon as possible and restored with appropriate treatment. Therefore, it is critical to have correct and early diagnosis of dysphagia in stroke patients.

Various studies have investigated dysphagia in relation to its incidence, patterns, and the effects of dysphagia therapy. Some of these studies described the characteristics of dysphagia patterns according to the location of brain lesions [4-12]. In many cases, however, it is difficult to clearly distinguish boundaries in brain lesions following a stroke. In addition, studies on the correlation between brain lesions and dysphagia symptoms often have different conclusions due to the presence of multifocal brain lesions. Most studies have categorized brain lesions according to cortical, subcortical, and brainstem locations, and their associated characteristic dysphagia patterns have been investigated for each lesion location $[3,6,7,9]$. Han et al. [2] conducted a study using a dysphagia scale, in which they examined the clinical characteristics of lesions in the middle cerebral artery (MCA) territory, basal ganglia, and thalamus. Some studies categorized lesions into frontal, parietotemporal, brainstem, and cerebellar locations to identify their clinical characteristics $[5,12]$. Additionally, some studies classified lesions based on their supratentorial location, and the correlation between lesion sites and dysphagia was investigated $[4,10]$.

This study investigated available cases after subdividing lesions into more specific categories. Groups with unilateral lesions were used to better demonstrate the related characteristics of the lesions. For clear identification of the areas with damage, this study was only conducted in patients with cerebral infarction. Unlike previous reports, this study aimed to investigate the characteristics of basal ganglia lesions, which are known to be a cause of dysphagia symptoms.

Some previous studies examined the correlation between dysphagia and brain lesions, but the number of patients was often insufficient for a significant statistical assessment. Moreover, these studies generally described dysfunction according to the anatomical location of each lesion. However, few studies conducted an objective evaluation to determine the component of swallowing function that was impaired. This study aimed to subdivide and compare the types of brain lesions that are commonly seen in patients with acute cerebral infarction, to enable an objective evaluation based on specific features of dysphagia, using a videofluoroscopic swallowing study (VFSS). Also dysphagia was evaluated with the videofluoroscopic dysphagia scale (VDS) for more insightful predictions and prognosis [2].

\section{MATERIALS AND METHODS}

\section{Study design and patients}

This was a retrospective study based on medical records. The study was conducted in patients who underwent VFSS from June 2013 to March 2017, after obtaining approval from the Institutional Review Board of DongEui Medical Center in Busan (No. DEMC-2017-13).

Of 4,940 stroke patients who visited the medical center during the study period, 275 survivors and other groups were included in the study. The inclusion criteria were all of the following: (1) patients had an acute stroke within the past 1 month; (2) patients had signs suggestive of dysphagia such as a reduced gag reflex, reduced laryngeal elevation, and coughing; and (3) patients had cerebral infarction.

The exclusion criteria were as follows: (1) inability to undergo tests and evaluation because of impaired mobility or cognitive deficits (Korean version of Mini-Mental State Examination [K-MMSE] score is less than 5 or one step obey not possible); (2) intracerebral hemorrhage; (3) bilateral lesions; (4) inadequate medical records; and (5) previous history of stroke.

For the purposes of this study, lesions were divided into 8 groups to compare VFSS results and conduct further tests (Fig. 1). The lesions were classified according to those frequently clinically observed and also to find out how the pattern of a swallowing disorder varies according to the location of the isolated lesion.

Table 1 presents the clinical information of the 275 patients and demographic characteristics of all lesion groups. The patients' diets were divided into three groups (tube feeding, dysphagia diet, regular diet) and the frequency and correlation with the lesions were confirmed.

There was a difference in diet between groups. The tube 

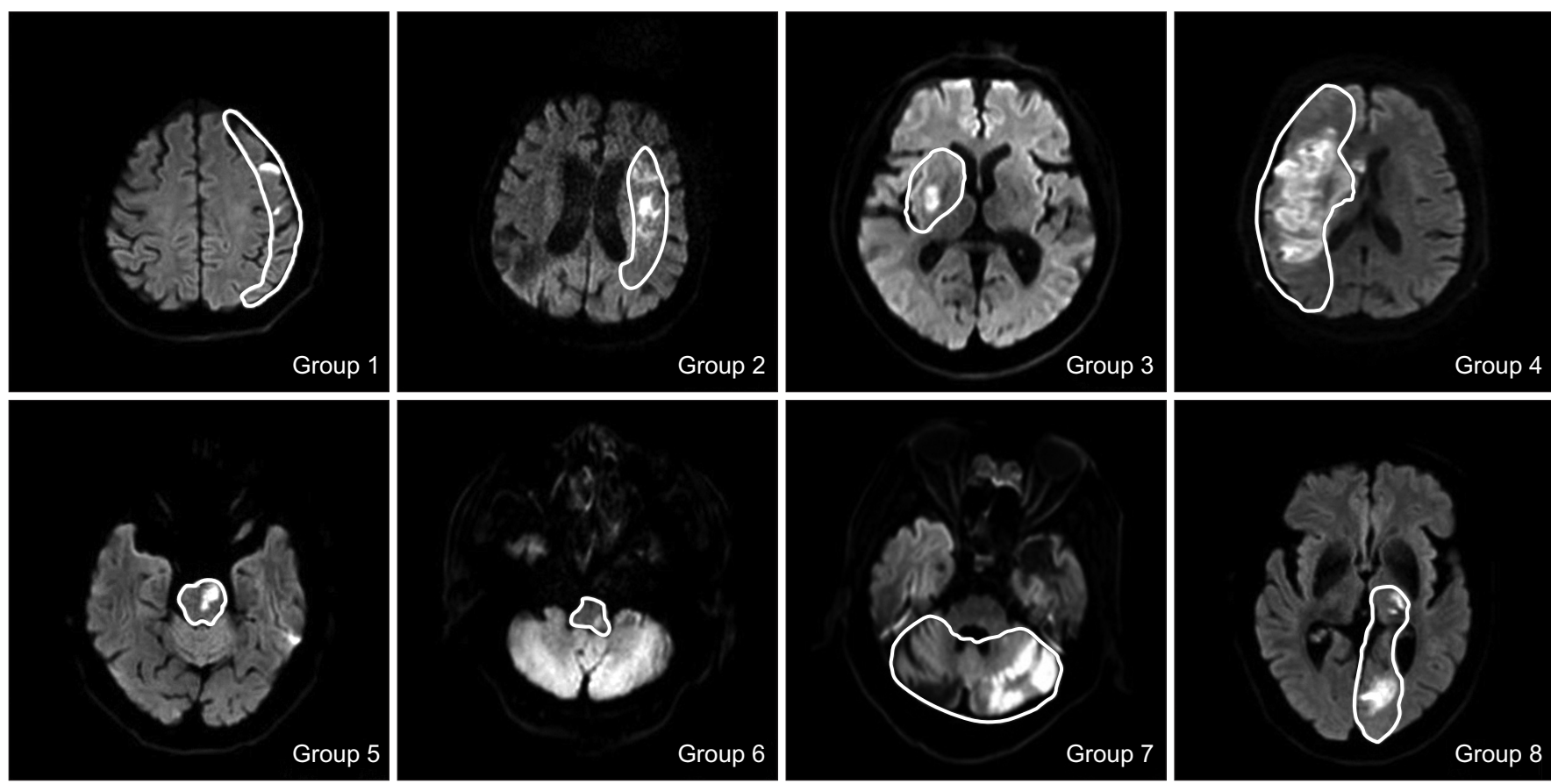

Fig. 1. The lesions were divided into 8 groups that can be observed clinically. The line represents the area of each group. Group 1, middle cerebral artery (MCA) territory (cortical lesions); Group 2, MCA territory (subcortical lesions except for basal ganglia lesions); Group 3, basal ganglia lesions; Group 4, MCA territory lesions, including basal ganglia lesions; Group 5, pontine lesions; Group 6, medullary lesions; Group 7, cerebellar lesions; Group 8, posterior cerebral artery (PCA) territory lesions.

feeding was higher in the 6 and 7 groups and the regular diet was lower in the 1 and 6 groups.

\section{Swallowing assessment}

To assess the 275 patients, VFSS was conducted according to a uniform protocol. VFSS was performed by physiatrists using a modification of the Logemann protocol [13] in radiography rooms in the radiology department. Tests were conducted with the patients in lateral position to better display the anatomical structures.

The patients were directed to swallow $2 \mathrm{~mL}$ of diluted barium twice, and an additional 5 or $10 \mathrm{~mL}$ of barium according to the investigator's discretion. Subsequently, identical tests were repeated using foods such as yogurt, puddings, rice porridge, and rice, with standardized viscosity and quality. The reference diet was pudding. But some subjects showed the lowest results in other diets and their results were based on the results of those diets.

\section{Outcome assessment}

We evaluated clinical dysphagia characteristics and VFSS findings in each patient group. With the VDS, dys- phagia severity can be quantitatively evaluated using a numerical scale (from 0 to 100). Lip closure, bolus formation, mastication, apraxia, tongue-to-palate contact, premature bolus loss, oral transit time, triggering of pharyngeal swallow, vallecular residue, laryngeal elevation, pyriform sinus residue, coating of the pharyngeal wall, pharyngeal transit time, and aspiration were evaluated [2]. By using quantified VDS scores, the 8 lesion groups were compared with each other to identify statistically significant items.

\section{Statistical methods}

Data were summarized using descriptive statistics as follows: frequency and percentage for categorical variables and mean \pm standard deviation or median (range) for continuous variables.

To investigate the differences among the 8 groups, the Kruskal-Wallis test with post-hoc Dunn's test was used. To check for a normal distribution, we used the ShapiroWilk test.

The effects of the independent variables on the response variables were analyzed using a multivariate lin- 


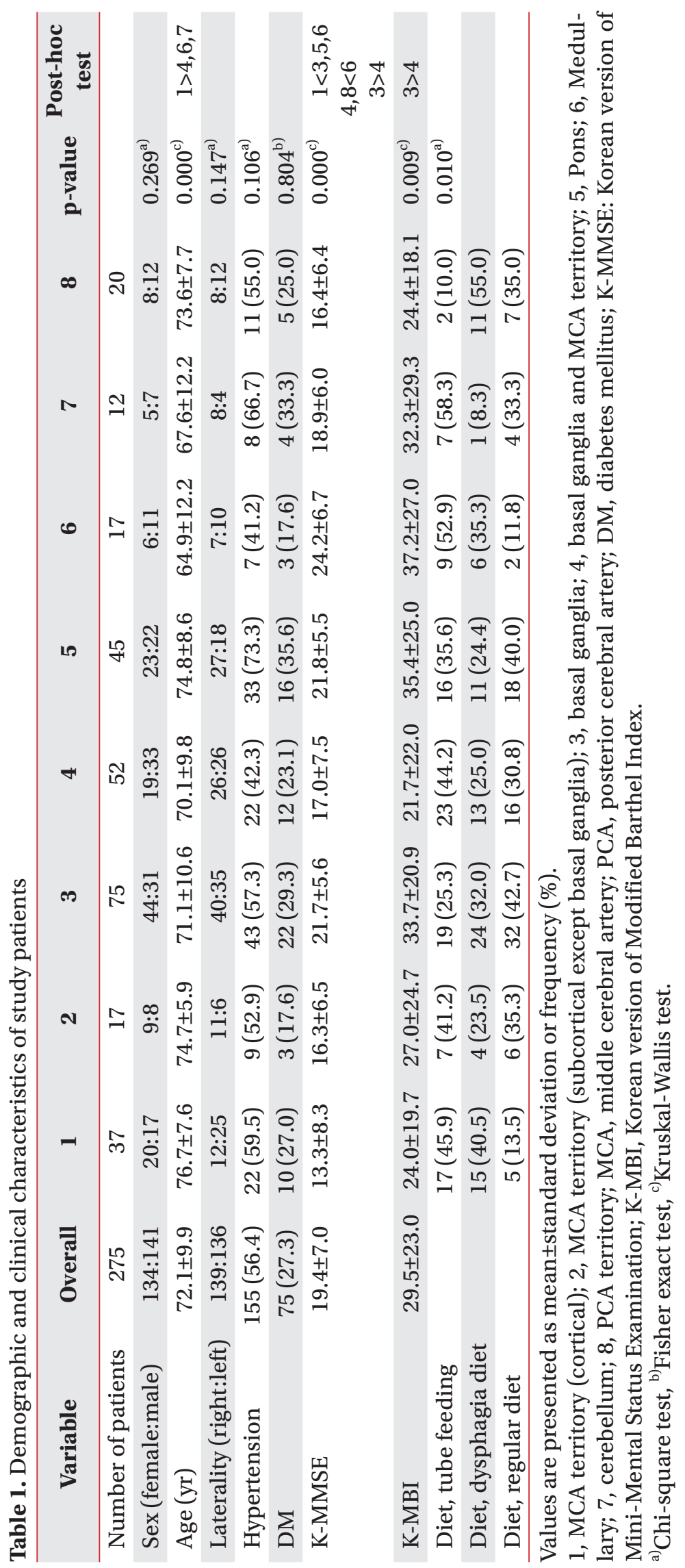


ear regression, and the statistically significant variables were selected using a backward elimination method with a 0.05 alpha level. To assess for multicollinearity, the variance inflation factor (VIF) was also estimated. VIF quantifies the severity of multicollinearity in a regression analysis, and a VIF of $<10$ indicates no problematic multicollinearity among the independent variables. We also set each VFSS finding as a dependent variable, and the corresponding odds ratio of each independent variable with a $95 \%$ confidence interval and equivalent $p$-value was calculated with the multivariate logistic regression analysis method. Variables were selected using a backward elimination method with a 0.05 alpha level.

The p-values of $<0.05$ were considered statistically significant. All statistical analyses were performed using SPSS version 22.0 for Windows (IBM Corp, Armonk, NY, USA) statistical software. All tests were two-tailed.

\section{RESULTS}

This study examined the correlation of each of the 8 lesion groups with the VDS scores obtained with a VFSS (Table 2). For cortical MCA territory lesions, apraxia was worse compared with other items. Mastication decreased more severely with subcortical MCA territory lesions than with pontine lesions. With basal ganglia lesions, pyriform sinus residue was greater than that in cortical MCA territory lesions. Also tongue-to-palate contact was more severe in basal ganglia lesions than subcortical MCA territory lesions.

Vallecular or pyriform sinus residue was comparatively more severe on pontine or medullary lesions than lesions in other sites. Coating of the pharyngeal wall deteriorated to a greater degree in posterior cerebral artery (PCA) territory lesions than in basal ganglia, pontine, medullary, and other lesions.

In addition, correlations with prognostic factors that influenced VFSS scores were identified (Table 3). Risk of dysphagia was relatively higher in males, and there was an inversely proportional correlation between the scores in the Korean version of the Modified Barthel Index (KMBI) and total VDS scores.

Evaluation of the relationships between subcategories of the VDS and prognostic factors identified similarities between VDS total scores and K-MBI scores. The outcome was more favorable when the K-MBI score was higher for lip closure, mastication, apraxia, laryngeal elevation, oral transit time, triggering of pharyngeal swallow, and pharyngeal transit time. In addition, males had greater pyriform sinus residue and worse pharyngeal transit times than females. Moreover, the pyriform sinus residue level increased with age (Table 4).

\section{DISCUSSION}

The subjects in this study were patients with acute cerebral infarction within the past one month who complained of dysphagia symptoms. Dysphagia according to brain lesion location was examined using more specific subcategories. Dysphagia was also examined for relationships with characteristic factors that may affect swallowing function.

In the past, it was assumed that swallowing disorders would not occur in unilateral hemisphere lesions, but only in bilateral hemisphere or brainstem lesions. This is because the brainstem, where cranial nerve nuclei and central neural circuits for swallowing reflexes are located, is controlled by both hemispheres. However, post-stroke dysphagia was observed in stroke patients with lateral or bilateral brainstem, and cortical and subcortical brain lesions $[14,15]$.

Pontine lesions can often cause oropharyngeal dysfunction, as the nucleus solitarius and nucleus ambiguus are located in the pons [6]. While patients with lateral pontine lesions typically display functional or nearly normal oral control, they have disorders involving triggering of neuromuscular control during pharyngeal phase swallowing. This could be due to significant delays in the triggering of pharyngeal phase swallowing, or to an increase in laryngeal upward movement and decrease in frontal movement, which leads to incomplete activation of the cricopharyngeus. Food residue may also be observed in the lateral pyriform sinuses. In this study, patient groups with this finding also showed significant results that indicated a disorder of the pharyngeal phase, such as vallecular residue or coating of the pharyngeal wall. Other studies suggested that the risk of pneumonia caused by aspiration is higher in patients with such lesions [16-18]. Despite the probability of pharyngeal dysfunction causing delayed aspiration, no significant result was observed in relation to aspiration and penetration on the VFSS.

Medullary lesions are usually accompanied by an in- 


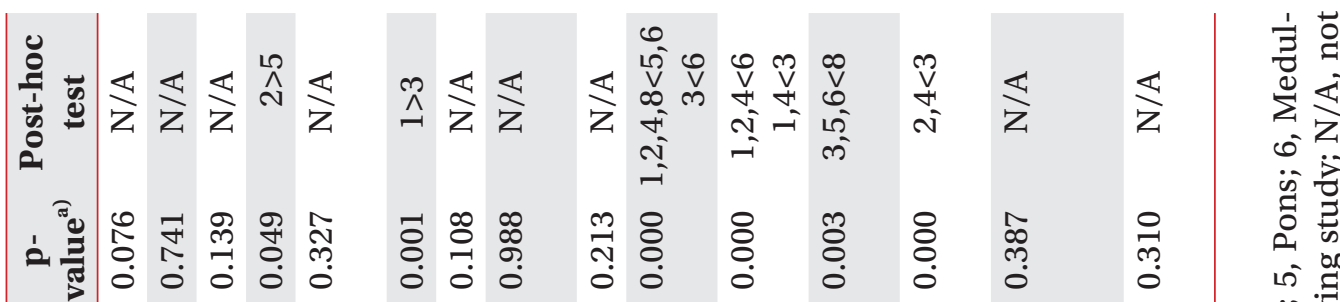

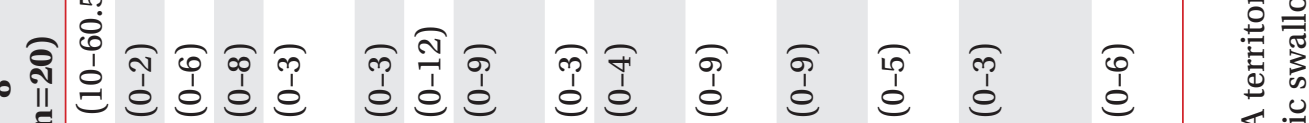

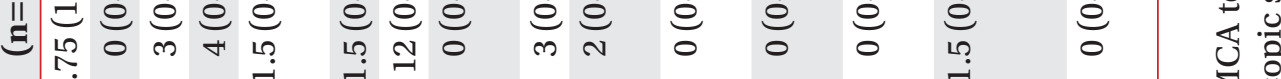
กั่

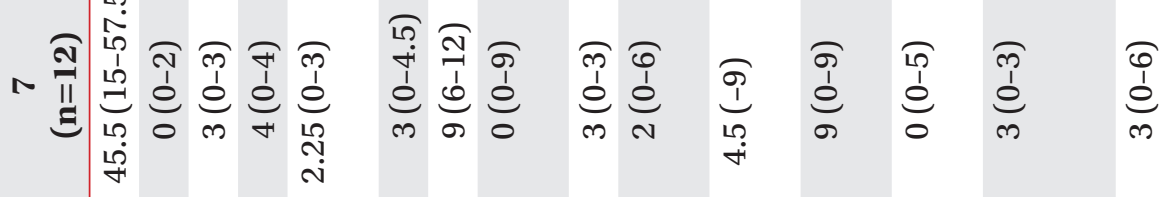

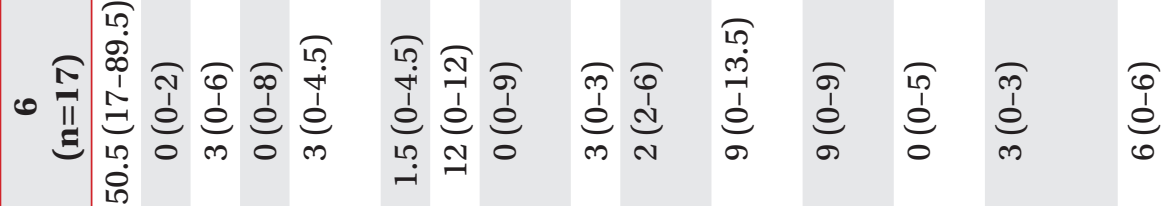

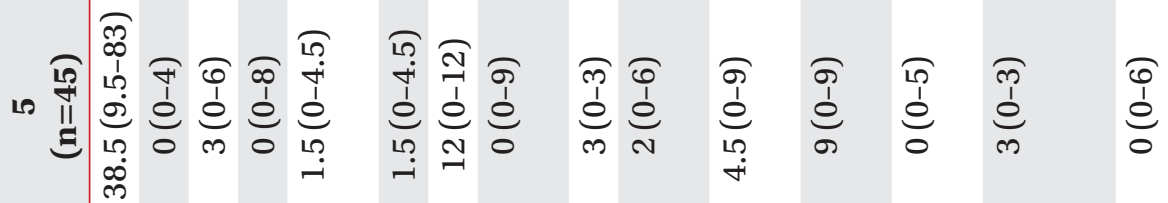

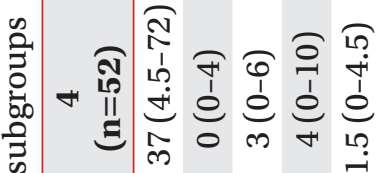

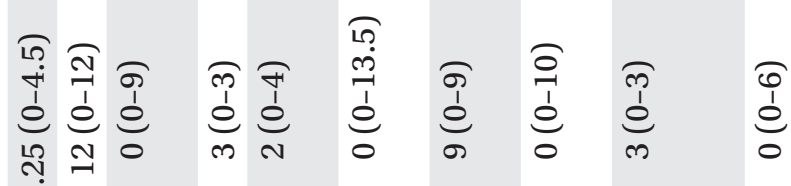

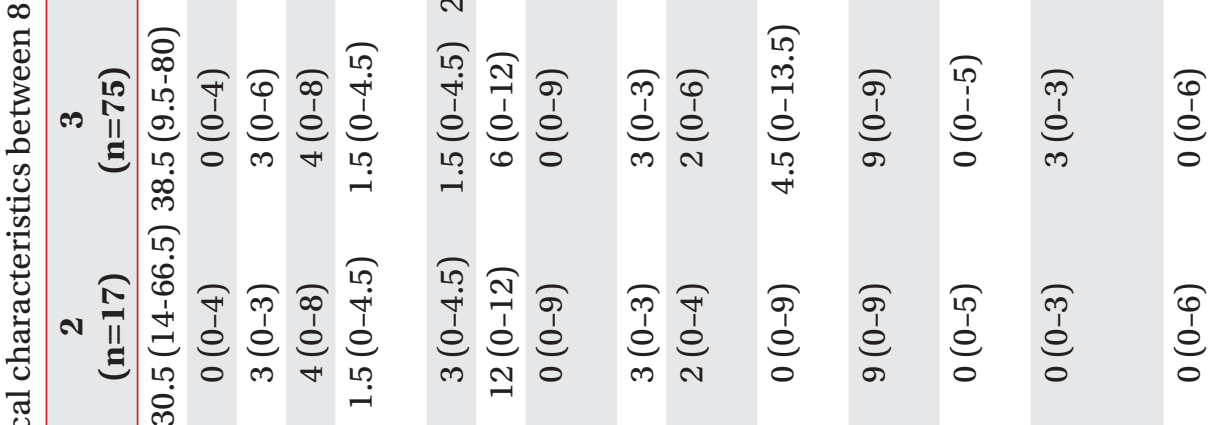

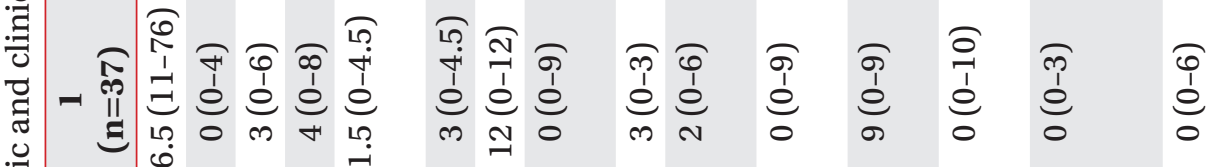
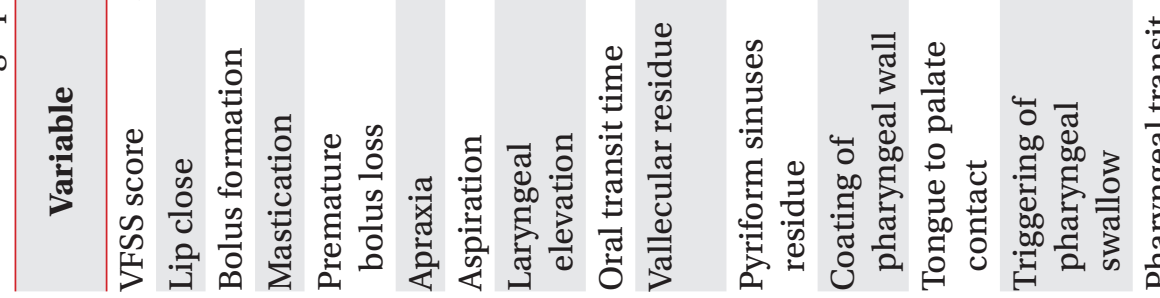
Table 3. Characteristic factor affecting VDS (VFSS) score (results from multivariate linear regression analysis)

\begin{tabular}{lccccc}
\hline \multicolumn{1}{c}{ Variable } & $\mathbf{B}$ & $\mathbf{9 5 \%} \mathbf{C I}$ & $\boldsymbol{\beta}$ & $\begin{array}{c}\text { p- } \\
\text { value }^{\text {a) }}\end{array}$ & VIF \\
\hline Sex (male) & 7.766 & 2.446 to 13.086 & 0.217 & 0.004 & 1.107 \\
Age & 0.127 & -0.146 to 0.400 & 0.071 & 0.359 & 1.163 \\
$\begin{array}{c}\text { Laterality } \\
\text { (right) }\end{array}$ & -2.281 & -7.613 to 3.051 & -0.063 & 0.400 & 1.098 \\
$\begin{array}{c}\text { Hypertension } \\
\text { (yes) }\end{array}$ & 1.267 & -3.943 to 6.478 & 0.035 & 0.632 & 1.038 \\
DM (yes) & -2.590 & -8.482 to 3.301 & -0.063 & 0.387 & 1.026 \\
K-MMSE & -0.105 & -0.529 to 0.319 & -0.041 & 0.627 & 1.381 \\
K-MBI & -0.138 & -0.258 to -0.017 & -0.182 & 0.025 & 1.273 \\
\hline
\end{tabular}

VDS, videofluoroscopic dysphagia scale; VFSS, videofluoroscopic swallowing study; $\mathrm{B}$, unstandardized regression efficient; CI, confidence interval; $\beta$, standardized regression coefficient; VIF, variance inflation index; DM, diabetes mellitus; K-MMSE, Korean version of Mini-Mental State Examination; K-MBI, Korean version of Modified Barthel Index.

${ }^{a)}$ Derived from multivariate linear regression analysis in Table 4. Sex, laterality, hypertension, and DM were treated as dummy variables. Reference category: sex (female $=0)$, laterality $(l e f t=0)$, hypertension $(\mathrm{no}=0)$, and $\mathrm{DM}$ $($ no $=0)$.

crease in swallowing muscle tension. Excessive muscle tension leads to delayed triggering of pharyngeal swallowing, loss of pharyngeal swallowing function, weakening or paralysis of the pharyngeal muscles, decreased laryngeal elevation, and impaired relaxation of the cricopharyngeus. Several studies have suggested that there should be no significant correlation between brain lesions and dysphagia. Falsetti et al. [19] reported finding no correlation between brainstem lesions and dysphagia. Other studies reported no significant relationship between the incidence of dysphagia and cerebellar lesions [20-22].

In this study, pharyngeal dysfunction was found to be prominent in subjects with medullary lesions, similar to the findings reported by Moon et al. [12]

In strokes involving the cerebral cortex, movement of the tongue itself is not significantly impaired, except when affected by apraxia, because the hypoglossal nucleus is located in the pons. Dysphagia symptoms can be associated with decreased pharyngeal peristalsis and delayed pharyngeal transit time [23]. This study also found that apraxia was significantly decreased in cortical MCA
Table 4. Factors affecting swallowing: multivariate prediction models (logistic regression analysis)

\begin{tabular}{lccc}
\hline \multicolumn{1}{c}{ Parameter } & OR & $\mathbf{9 5 \%}$ CI & $\begin{array}{c}\text { p- } \\
\text { value }^{\text {a) }}\end{array}$ \\
\hline $\begin{array}{l}\text { Lip close } \\
\text { K-MBI }\end{array}$ & 0.959 & $0.933-0.986$ & 0.003 \\
$\begin{array}{l}\text { Bolus formation } \\
\text { Laterality (right) }\end{array}$ & 0.421 & $0.197-0.898$ & 0.025 \\
$\begin{array}{l}\text { Hypertension (yes) } \\
\text { Mastication }\end{array}$ & 2.172 & $1.067-4.419$ & 0.032 \\
$\begin{array}{l}\text { K-MMSE } \\
\text { K-MBI }\end{array}$ & 0.950 & $0.905-0.998$ & 0.040 \\
$\begin{array}{l}\text { Premature bolus loss } \\
\quad \text { K-MBI }\end{array}$ & 0.985 & $0.971-0.999$ & 0.036 \\
$\begin{array}{l}\text { Apraxia } \\
\text { K-MBI }\end{array}$ & 0.987 & $0.975-1.000$ & 0.051 \\
\hline
\end{tabular}

Reduced laryngeal elevation and epiglottic closure
K-MBI
$\begin{array}{lll}0.981 & 0.966-0.997 & 0.020\end{array}$

Oral transit time

$\begin{array}{llll}\text { K-MBI } & 0.987 & 0.974-1.000 & 0.047\end{array}$

Residue on the valleculae

$\begin{array}{llll}\text { Laterality (right) } \quad 0.476 & 0.227-1.000 & 0.050\end{array}$

Residue in the pyriform

sinuses

$\begin{array}{llll}\text { Sex (male) } & 4.684 & 2.405-9.123 & 0.000 \\ \text { Age } & 1.037 & 1.003-1.072 & 0.034\end{array}$

Coating of pharyngeal wall

after swallow

Laterality (right) $\quad 0.520 \quad 0.269-1.006 \quad 0.052$

Pharyngeal delay time

K-MBI

$0.982 \quad 0.970-0.995 \quad 0.007$

Pharyngeal transit time

$\begin{array}{llll}\text { Sex (male) } & 2.029 & 1.089-3.782 & 0.026 \\ \text { K-MBI } & 0.986 & 0.972-0.999 & 0.038\end{array}$

Variable are selected using backward elimination method. OR, odds ratio; CI, confidence interval; DM, diabetes mellitus; K-MMSE, Korean version of Mini-Mental Status Examination; K-MBI, Korean version of Modified Barthel Index.

${ }^{a)}$ Derived from multivariate logistic regression analysis (regardless of severity, abnormal finding is set to 1 and normal to 0 ).

territory lesions.

Subcortical lesions affect not only the sensory pathways that communicate with the cerebral cortex, but also the 
motor pathways from the cortex. In addition, damage to the basal ganglia, including the corona radiata, frequently causes pharyngeal dysfunction [11]. Cerebral infarctions may display a mild delay in oral transit time and triggering of a swallowing reflex. The neuromuscular function involved in pharyngeal swallowing shows mild to moderate impairment. Some of these patients may experience aspiration before swallowing because of a delayed reflex or after swallowing because of impaired neuromuscular control [13]. To identify the differences between these lesions in detail, this study performed VFSS by categorizing subcortical lesions with or without basal ganglia involvement, and isolated basal ganglia lesions. However, there was no significant dysphagia pattern associated with the basal ganglia lesions.

Previous studies showed that swallowing dysfunction patterns differed according to lesion location in the left or right hemisphere. When the lesion was in the right hemisphere, the pharyngeal transit time was longer than when the lesion was in the left hemisphere. And pharyngeal dysfunction was more severe because of frequent aspiration and laryngeal infiltration. In contrast, patients with lesions in the left hemisphere showed impaired oral movements, including tongue movement disorders and delayed total pharyngeal reaction time $[24,25]$. In contrast with previous studies, this study observed no significant difference between groups with lesions in the left and right hemispheres.

This study also investigated the characteristic factors that may affect swallowing disorders. Dysphagia tended to be worse in males than in females. Patients with low K-MBI scores displayed more severe swallowing dysfunction. Moon et al. reported correlations between the KMMSE and the Korean version of the National Institutes of Health Stroke Scale scores with oral function [12]. This study demonstrated a significant relationship between the K-MMSE score and mastication, indicating a relationship between cognitive function and dysphagia. In our study, the inclusion criteria excluded subjects with highlevel dementia. Therefore, this limitation to our study may have affected the results.

In addition, several studies suggested that the assessment of a K-MBI should be an important factor in determining the prognosis of dysphagia $[19,26]$. These studies implied there were effects on prognostic factors such as cognitive function along with lesion location and accom- panying swallowing disorders $[19,27]$.

We could confirm dysphagia symptoms for each brain lesion, but there was no case for anterior cerebral artery (ACA) territory infarction lesions. This is because among the patients referred for swallowing by dysphagia, there were no patients with ACA territory infarction lesions included in this study with our inclusion criteria. The present study had some limitations. First, this was a retrospective analysis. Second, the classification of lesion location could have been more specific. Although we subdivided according to clinical features, lesion classification was subjectively determined by the investigator's discretion. Third, the study would have been more useful if the functional recovery and characteristics associated with the sub-items were investigated with a follow-up visit with patients who showed swallowing dysfunction. The clinical factors of patients were not totally controlled for in our study. In some groups, there was a difference in age and for some groups, other factors did not differ between groups. Also our study was conducted without consideration of the lesion volume and severity.

This study was significant in that it was conducted with a relatively large number of patients and involved patient groups with unilateral lesions. Moreover, the VDS was used to quantitatively evaluate the dysphagia sub-items. The results of this study can be used to predict lesionspecific parameters at the time of treatment for dysphagia, thereby enabling more accurate treatments. Additional studies on prognostic factors that may cause or exacerbate the disorders should be performed, to enable more accurate evaluation and treatment of dysphagia.

\section{CONFLICT OF INTEREST}

No potential conflict of interest relevant to this article was reported.

\section{REFERENCES}

1. Martino R, Foley N, Bhogal S, Diamant N, Speechley M, Teasell R. Dysphagia after stroke: incidence, diagnosis, and pulmonary complications. Stroke 2005;36:2756-63.

2. Han TR, Paik NJ, Park JW, Kwon BS. The prediction of persistent dysphagia beyond six months after stroke. Dysphagia 2008;23:59-64. 
3. Han H, Shin G, Jun A, Park T, Ko D, Choi E, et al. The relation between the presence of aspiration or penetration and the clinical indicators of dysphagia in poststroke survivors. Ann Rehabil Med 2016;40:88-94.

4. Suntrup S, Kemmling A, Warnecke T, Hamacher C, Oelenberg S, Niederstadt T, et al. The impact of lesion location on dysphagia incidence, pattern and complications in acute stroke. Part 1: dysphagia incidence, severity and aspiration. Eur J Neurol 2015;22:832-8.

5. Steinhagen V, Grossmann A, Benecke R, Walter U. Swallowing disturbance pattern relates to brain lesion location in acute stroke patients. Stroke 2009;40:1903-6.

6. Kim DK. Post-stroke dysphagia: incidence, complications and pattern relates to brain lesion. Brain Neurorehabil 2009;2:91-7.

7. Lee CK, Kim JA. Pattern of post-stroke swallowing disorder according to the brain lesion. J Korean Acad Rehabil Med 2001;25:193-201.

8. Dehaghani SE, Yadegari F, Asgari A, Chitsaz A, Karami $M$. Brain regions involved in swallowing: Evidence from stroke patients in a cross-sectional study. J Res Med Sci 2016;21:45.

9. Jeon WH, Park GW, Lee JH, Jeong HJ, Sim YJ. Association between location of brain lesion and clinical factors and findings of videofluoroscopic swallowing study in subacute stroke patients. Brain Neurorehabil 2014;7:54-60.

10. Kim BR, Moon WJ, Kim H, Jung E, Lee J. Association of dysphagia with supratentorial lesions in patients with middle cerebral artery stroke. Ann Rehabil Med 2016;40:637-46.

11. Jang S, Yang HE, Yang HS, Kim DH. Lesion characteristics of chronic dysphagia in patients with supratentorial stroke. Ann Rehabil Med 2017;41:225-30.

12. Moon HI, Pyun SB, Kwon HK. Correlation between location of brain lesion and cognitive function and findings of videofluoroscopic swallowing study. Ann Rehabil Med 2012;36:347-55.

13. Logemann JA. Evaluation and treatment of swallowing disorders. 2nd ed. Austin: Pro-Ed; 1998.

14. Michou E, Hamdy S. Cortical input in control of swallowing. Curr Opin Otolaryngol Head Neck Surg 2009;17:166-71.

15. Hamdy S, Mikulis DJ, Crawley A, Xue S, Lau H, Henry
S, et al. Cortical activation during human volitional swallowing: an event-related fMRI study. Am J Physiol 1999;277:G219-25.

16. DePippo KL, Holas MA, Reding MJ. The Burke dysphagia screening test: validation of its use in patients with stroke. Arch Phys Med Rehabil 1994;75:1284-6.

17. Gottlieb D, Kipnis M, Sister E, Vardi Y, Brill S. Validation of the $50 \mathrm{ml} 3$ drinking test for evaluation of poststroke dysphagia. Disabil Rehabil 1996;18:529-32.

18. Chua KS, Kong KH. Functional outcome in brain stem stroke patients after rehabilitation. Arch Phys Med Rehabil 1996;77:194-7.

19. Falsetti P, Acciai C, Palilla R, Bosi M, Carpinteri F, Zingarelli A, et al. Oropharyngeal dysphagia after stroke: incidence, diagnosis, and clinical predictors in patients admitted to a neurorehabilitation unit. J Stroke Cerebrovasc Dis 2009;18:329-35.

20. Langdon PC, Lee AH, Binns CW. Dysphagia in acute ischaemic stroke: severity, recovery and relationship to stroke subtype. J Clin Neurosci 2007;14:630-4.

21. Broadley S, Croser D, Cottrell J, Creevy M, Teo E, Yiu D, et al. Predictors of prolonged dysphagia following acute stroke. J Clin Neurosci 2003;10:300-5.

22. Paciaroni M, Mazzotta G, Corea F, Caso V, Venti M, Milia P, et al. Dysphagia following Stroke. Eur Neurol 2004;51:162-7.

23. Leonard R, Kendall K. Dysphagia assessment and treatment planning. 2nd ed. San Diego: Plural Publishing; 2008. p. 1-34.

24. Daniels SK, Foundas AL. Lesion localization in acute stroke patients with risk of aspiration. J Neuroimaging 1999;9:91-8.

25. Robbins J, Levine RL, Maser A, Rosenbek JC, Kempster GB. Swallowing after unilateral stroke of the cerebral cortex. Arch Phys Med Rehabil 1993;74:1295-300.

26. Broadley S, Cheek A, Salonikis S, Whitham E, Chong $\mathrm{V}$, Cardone D, et al. Predicting prolonged dysphagia in acute stroke: the Royal Adelaide Prognostic Index for Dysphagic Stroke (RAPIDS). Dysphagia 2005;20:303-10.

27. Toscano M, Cecconi E, Capiluppi E, Vigano A, Bertora P, Campiglio L, et al. Neuroanatomical, clinical and cognitive correlates of post-stroke dysphagia. Eur Neurol 2015;74:171-7. 